\title{
The contribution of red dwarfs and white dwarfs to the halo dark matter
}

\author{
S. Torres ${ }^{1,2}$, J. Camacho ${ }^{1}$, J. Isern ${ }^{3,2}$, and E. García-Berro ${ }^{1,2}$ \\ 1 Departament de Física Aplicada, Escola Politécnica Superior de Castelldefels, Universitat Politècnica de Catalunya, \\ Avda. del Canal Olímpic 15, 08860 Castelldefels, Spain \\ e-mail: gracia@fa.upc.es \\ 2 Institute for Space Studies of Catalonia, c/Gran Capità 2-4, Edif. Nexus 104, 08034 Barcelona, Spain \\ 3 Institut de Ciències de l'Espai, CSIC, Campus UAB, Facultat de Ciències, Torre C-5, 08193 Bellaterra, Spain
}

Received 18 March 2008 / Accepted 9 April 2008

\section{ABSTRACT}

\begin{abstract}
Context. The nature of the several microlensing events observed by the MACHO team towards the LMC still remains controversial. Low-mass substellar objects and stars with masses larger than $\sim 1 M_{\odot}$ have been ruled out as major components of a MACHO Galactic halo, while stars of half solar masses are the most probable candidates.

Aims. We assess jointly the relative contributions of both red dwarfs and white dwarfs to the mass budget of the Galactic halo. Methods. We use a Monte Carlo simulator that incorporates up-to-date evolutionary sequences of both red dwarfs and white dwarfs as well as detailed descriptions of both our Galaxy and the LMC. We explore a complete mass range between 0.08 and $1 M_{\odot}$ of possible microlensing candidates and we compare the synthetic populations obtained with our simulator with the results obtained by the MACHO and EROS experiments.

Results. The contribution of the red-dwarf population is insufficient to explain the number of events claimed by the MACHO team; this is even though the optical depth measured for the white-dwarf population alone, increases by a factor of two, after the addition of the red-dwarf population.

Conclusions. We find that the contribution to the halo dark matter of the entire population under study, is smaller than $10 \%$, at the $95 \%$ confidence level.
\end{abstract}

Key words. stars: luminosity function, mass function - stars: white dwarfs - Galaxy: halo - stars: low-mass, brown dwarfs - Galaxy: stellar content - galaxies: structure

\section{Introduction}

Several cosmological observations show compelling evidence that baryons represent only a small fraction of the total matter in our Universe and that non-baryonic dark matter dominates over baryons. Within the standard cosmological model, this naturally leads to the existence of a type of unknown energy, the dark energy, which is the predominant component, $\Omega_{\Lambda} \simeq 0.72$, and a dark-matter component, for which $\Omega_{\mathrm{M}} \simeq 0.27$, in contrast to the baryonic component, for which $\Omega_{\mathrm{B}} \simeq 0.044$. Moreover, most of the baryons are non-luminous, since the density of luminous matter is $\Omega_{\star} \simeq 0.005$. In the case of the Galaxy, it was found that the virial mass, out to $100 \mathrm{kpc}$, is $M \approx 10^{12} M_{\odot}$, while the baryonic mass in the form of stars is $M_{\star} \approx 7 \times 10^{10} M_{\odot}$, which implies that the baryon fraction of the Galaxy is at most $8 \%$ (Klypin et al. 2007). This paradox is known as the missing-baryon problem - see the review of Silk (2007) for a complete, interesting discussion of this issue - and its resolution is critical to our understanding of how the Galaxy (and, in addition, by extension other galaxies) were formed and subsequently evolve. Three alternative solutions can be envisaged: (i) these baryons are in the outer regions of our Galaxy; (ii) they were never present in the protogalaxy; or (iii) they may have been ejected from the Milky Way. The most-promising explanation, and presently the most favored one, is the first of these options.

Since the pioneering proposal of Paczyński (1986) that gravitational microlensing could be used to clarify the nature of
Galactic dark matter, considerable observational and theoretical efforts have been invested in this issue. Among the most likely candidates for building-up the baryonic, dark-matter density are massive, baryonic halo-objects, or MACHOs. From the theoretical point of view it has been suggested that MACHOs could be planets $\left(M \sim 10^{-7} M_{\odot}\right)$, brown and red dwarfs (with masses ranging from $\sim 0.01$ to $\sim 1 M_{\odot}$ ), primordial black holes $\left(M \gtrsim 10^{-16} M_{\odot}\right)$, molecular clumps $\left(M \sim 1 M_{\odot}\right)$, and old white dwarfs $\left(M \sim 0.6 M_{\odot}\right)$. From the observational perspective, the MACHO (Alcock et al. 1997, 2000), EROS (Lasserre et al. 2001; Goldman et al. 2002; Tisserand et al. 2007), OGLE (Udalski et al. 1994), MOA (Muraki et al. 1997), and SuperMACHO (Becker et al. 2005) teams have monitored millions of stars during several years, in both the Large Magellanic Cloud (LMC) and the Small Magellanic Cloud (SMC), to search for microlensing events. However, only the MACHO team and the EROS group have reported their results. Although differences were initially found between the results, agreement has now been reached in some of the most important points. For instance, no microlensing candidates were found by the MACHO team or the EROS group with event durations between a few hours and 20 days. This implied that the Galactic halo could contain no more than a $10 \%$ of dark objects in the mass range $10^{-7}<M / M_{\odot}<$ $10^{-3}$. This ruled out planets and brown dwarfs as the major contributors to the mass budget of the dark halo. Moreover, the MACHO collaboration detected $\sim 15$ microlensing events during their $5.7 \mathrm{yr}$ analysis of 11.9 million stars in the LMC 
(Alcock et al. 2000). In their analysis, they derived an optical depth towards the LMC of $\tau=1.2_{-0.3}^{+0.4} \times 10^{-7}$ or, equivalently, a halo fraction $0.08<f<0.50$, at the $95 \%$ confidence level, with a MACHO mass in the range $0.15 \leq M / M_{\odot} \leq 0.50$, depending on the halo model. On the other hand, the non-detections reported by the EROS collaboration provided an upper limit. The EROS team presented an analysis of a subsample of bright stars belonging to the LMC (Tisserand et al. 2007), to minimize source confusion and blending effects. Their results implied that the optical depth towards the LMC was $\tau<0.36 \times 10^{-7}$, at the $95 \%$ confidence level, corresponding to a fraction of the halo mass of less than $7 \%$. This result was 4 times smaller than that obtained by the MACHO team. Consequently, this started discussion about the location and nature of the lenses. In particular, LMC models were used to ascertain if possible asymmetries in the space distribution of the microlensed stars could be related to Galactic halo objects or LMC ones. In fact, different studies indicate that a sizeable fraction of the microlensed events are due to LMC self-lensing (Sahu 1994; Gyuk et al. 2000; Calchi Novati et al. 2006). Moreover, a full variety of possible explanations were proposed to reproduce the microlensing events: tidal debris or a dwarf galaxy toward the LMC (Zhao 1998), a Galactic-extended shroud population of white dwarfs (Gates \& Gyuk 2001), blending effects (Belokurov et al. 2003, 2004), spatially-varying mass-functions (Kerins \& Evans 1998; Rahvar 2005), and other explanations (Holopainen et al. 2006). However, all of these proposals were received with some criticism because none fully explained the observed microlensing results.

Other observational pieces of evidence were added to help resolve the present puzzle, such as the search for very faint objects in the Hubble Deep Field or the search for the microlensing events towards the Galactic bulge or towards very crowded fields such as M 31. In particular, the Hubble Deep Field provided an opportunity to test the contribution of white dwarfs to the Galactic dark matter. Ibata et al. (1999) and Kilic et al. (2005) claimed the detection of some white-dwarf candidates among several faint blue objects. These objects exhibit significant proper motion and are, thus, assumed to belong to the thick disk or the halo populations. Despite the increasing number of surveys that are searching for white dwarfs - such as the Sloan Digital Sky Survey (Eisenstein et al. 2006), the 2 Micron All Sky Survey (Cutri et al. 2003), the SuperCosmos Sky Survey (Hambly et al. 2001), the 2dF QSO Redshift Survey (Vennes et al. 2002), and other observational searches (Knox et al. 1999; Ibata et al. 1999; Oppenheimer et al. 2001; Majewski \& Siegel 2002; Nelson et al. 2002) - and the numerous theoretical studies (Isern et al. 1998; Reylé et al. 2001; Flynn et al. 2003; Hansen \& Liebert 2003; García-Berro et al. 2004), the problem still remains open. Despite the fact that, for obvious reasons, no information about the halo could be derived from the microlensing events towards the Galactic bulge, the results obtained so far indicate the primordial role played by low-mass stars rather than other objects.

In two previous papers, we extensively analyzed the role played by the carbon-oxygen (CO) white-dwarf population (García-Berro et al. 2004) in addition to the contribution of oxygen-neon (ONe) white dwarfs (Camacho et al. 2007). We performed a thorough study of a wide range of Galactic inputs, including different initial mass functions and halo ages, and several density profiles corresponding to different halo models. The calculations indicated that a sizeable fraction of the halo dark matter cannot be locked in the form of white dwarfs. We found that the contribution of white dwarfs is, in fact, approximately a modest $5 \%$ in the most optimistic case. This contribution is mainly due to old CO white dwarfs with hydrogen-rich atmospheres and the contribution of $\mathrm{ONe}$ white dwarfs is minor, because although $\mathrm{ONe}$ white dwarfs can reach faint magnitudes more rapidly than $\mathrm{CO}$ white dwarfs, their contribution is heavily suppressed by the initial mass function.

In this paper, we analyze, in a comprehensive way, a significant range of masses $0.08<M / M_{\odot}<10$ likely to produce microlensing events towards the LMC and, thus, to contribute to the halo dark matter. This mass range represents almost $90 \%$ of the stellar content, including the red-dwarf regime $\left(M>0.075 M_{\odot}\right)$, the $\mathrm{CO}$ white-dwarf population, and the population of massive ONe white dwarfs. The paper is organized as follows. In Sect. 2. we summarize the main ingredients of our Monte Carlo code and other basic assumptions and procedures necessary to evaluate the microlensing optical depth towards the LMC. Section 3 is devoted to describing our main results, including the contribution of red dwarfs and white dwarfs to the microlensing optical depth towards the LMC, and we compare our results to those of the MACHO and EROS teams. In this section, we also estimate the probability that a microlensing event could be assigned to one or another of the populations under study and we discuss the contribution of red and white dwarfs to the baryonic content of the Galaxy. In Sect. 4, our we summarize our major findings and draw our conclusions.

\section{The model}

\subsection{Building the sample}

A detailed description of our Monte Carlo simulator was presented in Torres et al. (2002), García-Berro et al. (2004), and Camacho et al. (2007). We summarize its most important features and inputs. The basic ingredient of any Monte Carlo simulator is a random-number-generator algorithm that must ensure a non-correlated sequence and a good set of statistical properties. We used a random-number-generator algorithm (James 1990) that provides a uniform probability density within the interval $(0,1)$ and ensures a repetition period of $\gtrsim 10^{18}$, which is sufficient for our purposes. Each one of the Monte Carlo simulations presented here consists of an ensemble of $\sim 5 \times 10^{4}$ independent realizations of the synthetic-star population, for which the average of any observational quantity, along with its corresponding standard deviation, were computed. Here the standard deviation means the ensemble mean of the sample dispersions for a typical sample.

The Galactic halo was modeled assuming a sphericallysymmetric halo. In particular, the model used here is an isothermal sphere of radius $5 \mathrm{kpc}$ also referred to as the "S-model", which was used extensively by the MACHO collaboration (Alcock et al. 2000; Griest 1991). The position of each synthetic star was randomly chosen according to this density profile. We have not used other profiles, such as the exponential power-law model, the Navarro et al. (1997) density profile, and others, because their inclusion would not have significantly changed the final results (García-Berro et al. 2004). This reasoning is also applicable to the non-standard Galactic halo models, such as flattened profiles, oblate halo models, and others, which are beyond the scope of the present study.

The mass distribution of synthetic stars was computed using two different initial mass functions, the standard initial mass function of Scalo (1998) and the biased log-normal initial mass function proposed by Adams \& Laughlin (1996), the latter being representative of other non-conventional initial mass 
functions, such as that of Chabrier et al. (1996). We note, however, that these biased initial mass functions appear to be incompatible with the observed properties of the halo white-dwarf population (Isern et al. 1998; García-Berro et al. 2004), with the contribution of thermonuclear supernovae to the metallicity of the Galactic halo (Canal et al. 1997), and with the observations of galactic halos in deep galaxy surveys (Charlot \& Silk 1995). For the sake of completeness we prefer, however, to include a representative example of these biased mass functions to illustrate the role played by the red dwarf population when a biased initial mass function is adopted.

The main-sequence mass is obtained by drawing a pseudorandom number, according to the adopted initial mass function. The time at which a star was born is randomly choosen, previously assuming that the halo was formed 14 Gyr ago in an intense burst of star formation of duration $\sim 1$ Gyr. The mainsequence lifetime, as a function of the mass in the main sequence, is that of Iben \& Laughlin (1989). As soon as the mass of a synthetic star is chosen, its main-sequence lifetime is inferred and we are the able to determine which stars are able to evolve into white dwarfs or remain on the main sequence as red dwarfs. We considered red dwarfs to have masses in the range $0.08<M / M_{\odot}<1$. For these stars, we adopted the evolutionary models of Baraffe et al. (1998). Stars with such small masses have large main-sequence lifetimes; no post-mainsequence evolutionary tracks of these stars were, therefore, required. Effective temperatures, luminosities, and colors were derived for stars that had time to enter the white-dwarf cooling track, given a set of theoretical cooling-sequences and an initialto-final-mass relationship (Iben \& Laughlin 1989). The cooling sequences adopted here depend on the mass of the white dwarf. White dwarfs with masses smaller than $M_{\mathrm{WD}}=1.1 M_{\odot}$ are expected to have a CO core and for these we adopt the cooling tracks of Salaris et al. (2000). White dwarfs with masses larger than $M_{\mathrm{WD}}=1.1 M_{\odot}$ probably have ONe cores; for these white dwarfs, we adopt the cooling sequences of Althaus et al. (2007). Both sets of cooling sequences incorporate the most accurate physical inputs for the stellar interior (including neutrinos, crystallization, phase separation and Debye cooling) and reproduce the blue turn at low luminosities (Hansen 1998).

The kinematical properties of the halo population were modeled using Gaussian laws (Binney \& Tremaine 1987) with radial and tangential velocity dispersions that were related by the Jean's equation to reproduce the flat rotation curve of our Galaxy. We adopted standard values for the circular velocity $V_{\mathrm{c}}=220 \mathrm{~km} \mathrm{~s}^{-1}$, in addition to the peculiar velocity of the Sun $\left(U_{\odot}, V_{\odot}, W_{\odot}\right)=(10.0,15.0,8.0) \mathrm{km} \mathrm{s}^{-1}$ (Dehnen \& Binney 1998). We discarded stars that had velocities smaller than $250 \mathrm{~km} \mathrm{~s}^{-1}$, which were not considered as halo members. We also rejected stars of velocities larger than $750 \mathrm{~km} \mathrm{~s}^{-1}$, because these velocities exceeded 1.5 times the escape velocity. Since white dwarfs do not usually have radia-component determinations of their velocities, the radial velocity of each model star is not considered when a comparison with observational data is completed.

To compare simulated results with observations, a normalization criterion should be used. We proceeded as in our previous papers (Camacho et al. 2007; García-Berro et al. 2004), that is, we normalized our simulations to the local density of halo white dwarfs obtained from the halo white dwarf luminosity function of Torres et al. (1998), we also took account the new halo white-dwarf candidates in the SDSS Stripe 82 (Vidrih et al. 2007).

\subsection{The LMC microlensing model}

To reproduce the microlensing experiments towards the LMC, we followed the detailed LMC descriptions of Gyuk et al. (2000) and Kallivayalil et al. (2006). Our model takes account of parameters such as the scale length and scale height of the LMC, its inclination, and its kinematical properties. This model provides a synthetic population of stars representative of the monitored point sources. After each simulation was completed, we evaluated which star of the Galactic halo could be responsible for a microlensing event. We considered only stars that fulfilled a series of criteria. First, the lensing star should be fainter than a certain magnitude limit. In a second step, we checked whether the lens was inside the Einstein tube of the monitored star, that is, if the angular distance between the lens and monitored star was smaller than the Einstein radius. We recall here that the Einstein radius is given by

$R_{\mathrm{E}}=2 \sqrt{\frac{G M D_{\mathrm{OS}}}{c^{2}} x(1-x)}$

where $D_{\mathrm{OS}}$ is the observer-source distance and $x \equiv D_{\mathrm{OL}} / D_{\mathrm{OS}}$. Third, we filter those stars that are candidates to produce a microlensing event with the detection efficiency function, $\varepsilon\left(\hat{t}_{i}\right)$, where $\hat{t}_{i}$ is the Einstein ring diameter crossing time. The detection efficiency depends on the particular characteristics of the experiment. We reproduced the MACHO and EROS experiments. In the case of the MACHO project, we adopted $1.1 \times 10^{7}$ stars during $5.7 \mathrm{yr}$ over $13.4 \mathrm{deg}^{2}$, whereas the detection efficiency was modeled according to:

$\varepsilon(\hat{t})=\left\{\begin{array}{cc}0.43 \mathrm{e}^{-\left(\ln \left(\hat{t} / T_{\mathrm{m}}\right)\right)^{3.58} / 0.87}, & \hat{t}>T_{\mathrm{m}} \\ 43 \mathrm{e}^{-\left|\ln \left(\hat{t} / T_{\mathrm{m}}\right)\right|^{2.34} / 11.16}, & \hat{t}<T_{\mathrm{m}}\end{array}\right.$

where $T_{\mathrm{m}}=250$ days. This expression provides a good fit to the results of Alcock et al. (2000). For the EROS experiment, we used $0.7 \times 10^{7}$ stars over a wider field of $84 \mathrm{deg}^{2}$ and a period of $6.7 \mathrm{yr}$. To evaluate the detection efficiency, we adopted a numerical fit to the results of Tisserand et al. (2007).

For all of the simulations, we extract parameters required to characterize the microlensing experiments. A complete description of parameters important to a discussion of gravitational microlensing can be found in Mollerach \& Roulet (2002) and Schneider et al. (2004). The most important parameter, for our purposes, is probably the optical depth, $\tau$, which measures the probability that, at a given time, a star is magnified by a lens by more than a factor of 1.34. Observationally, an estimate of this parameter can be obtained using the expression (Alcock et al. 2000):

$\tau=\frac{1}{E} \frac{\pi}{4} \sum_{i} \frac{\hat{t}_{i}}{\varepsilon\left(\hat{t}_{i}\right)}$

where $E$ is the total exposure in star-years. The optical depth is independent of both the lens motion and mass distribution. Since the experiments measure the number of events and their durations, additional information can be however obtained using the microlensing rate, $\Gamma$, and its distribution as a function of event duration. The microlensing rate represents the flux of lenses inside the microlensing tube. Finally, an estimate of the expected number of events can be evaluated using the expression

$N_{\exp }=E \int_{0}^{\infty} \frac{\mathrm{d} \Gamma}{\mathrm{d} \hat{t}} \varepsilon\left(\hat{t}_{i}\right) \mathrm{d} \hat{t}_{i}$ 


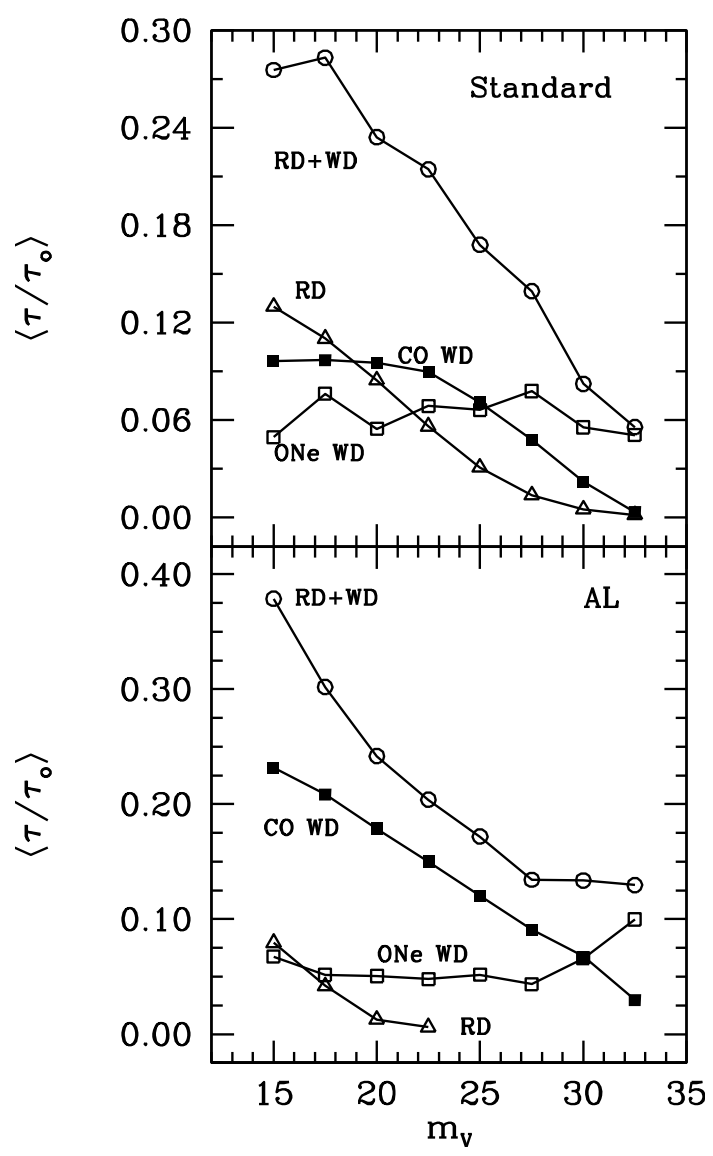

Fig. 1. Microlensing optical depth towards the LMC as a function of the limiting magnitude. Solid and open squares represent the $\mathrm{CO}$ and ONe white-dwarf populations, respectively. Red dwarfs are represented using open triangles, while the entire population is shown using open circles.

\section{Results}

\subsection{Optical depth towards the LMC}

As previously mentioned, the optical depth provides the most obvious and basic information about the microlensing experiments. Even so, the optical depth plays a critical role in our analysis, since it provides a wealth of information about the Galactic halo and the presence of dark matter. We compare results obtained using our Monte Carlo simulator with those derived by the MACHO collaboration. In Fig. 1, we present the contribution of the different simulated populations, to the optical depth, for the two initial mass functions studied, as a function of the adopted magnitude cut. Our simulations were normalized to the value of the optical depth derived by Alcock et al. (2000), $\tau_{0}=1.2 \times 10^{-7}$. The white-dwarf populations are represented by solid and open squares for the $\mathrm{CO}$ and $\mathrm{ONe}$ white dwarfs, respectively. Open triangles indicate the contribution of red dwarfs, while the contribution of the three populations is represented by open circles. The first remarkable result is that, for the standard initial mass function (top panel of Fig. 1), the combined contribution of red dwarfs and white dwarfs is at most one third of the observed optical depth, when a totally-unrealistic magnitude cut is adopted, $m_{V} \sim 15^{\mathrm{mag}}$. We note, in addition, that there is a clearly-decreasing trend as the magnitude-cut adopted is increased. This is because fewer objects contribute to the optical depth measured as the magnitude cut increases. Furthermore, the slope of the distributions is different for the three types of objects considered. For instance, the contribution of red dwarfs decreases faster than the contribution of $\mathrm{CO}$ white dwarfs as the magnitude cut increases, which, in turn, decreases faster than the contribution of ONe white dwarfs. This reflects the fact that, in general, red dwarfs are brighter than regular, $\mathrm{CO}$ white dwarfs. Finally, ONe white dwarfs cool rapidly (Althaus et al. 2007) and thus, for realistic halo ages, are faint objects. Consequently, their contribution remains almost constant. We note that the optical depth, measured for the entire population, almost doubles that obtained when the white-dwarf population alone is considered. The value measured remains, however, far below the observed value, which agrees with other studies; it is clear that the mass of a Galactic halo population cannot fully explain the results of the MACHO team. Even for different initial mass functions, for example that of Adams \& Laughlin (1996), we are able to predict that $\left\langle\tau / \tau_{0}\right\rangle$ is, at most, 0.3 , which is evaluated for unrealistic magnitude cuts (see the bottom panel of Fig. 1). The results obtained using the non-standard initial mass function deserve two additional comments. In particular, we note that the largest contribution to the optical depth measurement is made by the $\mathrm{CO}$ white-dwarf population and, secondly, that the contribution of red dwarfs is negligible for magnitude cuts larger than $m_{V} \sim 23^{\mathrm{mag}}$, which is a reasonable value for current surveys. Both facts are unsurprising, since this particular initial mass function was tailored to produce a large population of $0.5 M_{\odot}$ white dwarfs. In summary, we find that, for the mass range considered, a model stellar halo produces a microlensing optical-depth that is at most one third of that measured by the MACHO team, independently of the adopted initial mass function.

In Table 1, we summarize the average values of parameters of the entire population for two initial mass functions and four magnitude cuts, that is, we present the number of microlensing events, the average mass of the microlenses, their average proper motion, distance and tangential velocity, the corresponding Einstein crossing times and, finally, the contribution to the microlensing optical depth. It is clear that some parameters are dependent of the magnitude cut. For instance, the average distance of the sample increases as the magnitude cut increases. This is a natural consequence of selecting more distant objects which, in turn, implies longer Einstein crossing times. This behavior is independent of the assumed initial mass function. However, there are characteristics which do not change as the magnitude cut increases, for instance, the expected number of events or the average mass of the sample. In the case of a standard initial mass function, no more than one microlensing event should be expected at the $1 \sigma$ confidence level, while for a lognormal mass function up to 5 events might be expected. In any case, the expected number of microlensing events is far from the 17 events claimed by the MACHO experiment.

As already mentioned, the average mass of the microlenses depends on the assumed initial mass function. To investigate this further in Fig. 2, the contribution of the microlenses to the optical depth as a function of the mass of the lens object, for both initial mass functions, is shown. The results obtained with our Monte Carlo simulator clearly show that for a standard initial mass function (shown in the top panel of Fig. 2) there are two peaks centered at masses $\sim 0.3 M_{\odot}$ and $\sim 0.6 M_{\odot}$, respectively. These masses correspond to the average masses of red dwarfs and $\mathrm{CO}$ white dwarfs, respectively. We note that, in the case of a standard initial mass function, the contribution of ONe white dwarfs appears as an extended tail in the case of a standard initial mass function. This agrees with studies of the distribution of masses of the white-dwarf population (Finley et al. 1997; 
Table 1. Summary of the results obtained for the entire simulated population of microlenses in the direction of the LMC for a halo age of 14 Gyr, different model initial mass functions, and several magnitude cuts.

\begin{tabular}{lrrrrrrrr}
\hline \hline & \multicolumn{3}{c}{ Standard } & \multicolumn{4}{c}{ AL } \\
\hline Magnitude & 17.5 & 22.5 & 27.5 & 32.5 & 17.5 & 22.5 & 27.5 & 32.5 \\
\cline { 2 - 8 }$\left\langle N_{\mathrm{WD}}\right\rangle$ & $0 \pm 1$ & $0 \pm 1$ & $0 \pm 1$ & $0 \pm 1$ & $3 \pm 2$ & $2 \pm 1$ & $1 \pm 1$ & $0 \pm 1$ \\
$\langle m\rangle\left(M / M_{\odot}\right)$ & 0.421 & 0.411 & 0.427 & 0.443 & 0.638 & 0.636 & 0.640 & 0.684 \\
$\langle\mu\rangle\left({ }^{\prime \prime} \mathrm{yr}^{-1}\right)$ & 0.020 & 0.017 & 0.008 & 0.004 & 0.036 & 0.025 & 0.011 & 0.003 \\
$\langle\mathrm{~d}\rangle(\mathrm{kpc})$ & 2.48 & 3.79 & 6.62 & 13.08 & 1.39 & 2.15 & 5.13 & 19.6 \\
$\left\langle V_{\text {tan }}\right\rangle\left(\mathrm{km} \mathrm{s}^{-1}\right)$ & 240 & 247 & 262 & 241 & 240 & 252 & 263 & 261 \\
$\left\langle\hat{t}_{\mathrm{E}}\right\rangle(\mathrm{d})$ & 41.2 & 49.3 & 63.3 & 82.8 & 34.7 & 46.6 & 76.4 & 126.8 \\
$\left\langle\tau / \tau_{0}\right\rangle$ & 0.283 & 0.214 & 0.139 & 0.055 & 0.302 & 0.204 & 0.140 & 0.129 \\
\hline
\end{tabular}

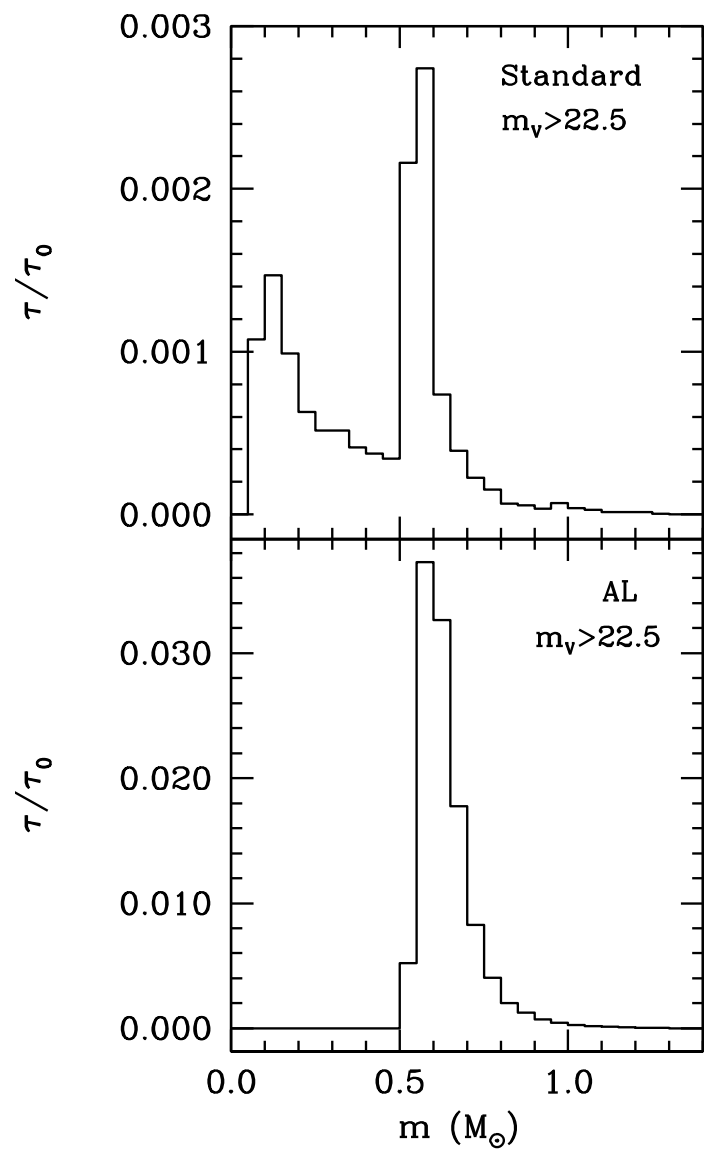

Fig. 2. Contribution to the optical depth as a function of lens mass.

Liebert et al. 2005). These studies indicate that a narrow, sharp peak exists close to $0.6 M_{\odot}$, with a tail extending towards larger masses, and that several white dwarfs with spectroscopicallydetermined masses occupy the mass interval between 1.0 and $1.2 M_{\odot}$. The situation is different for the non-standard initial mass function, which is shown in the bottom panel of Fig. 2. The log-normal initial mass function considered here cannot produce red dwarfs with masses below $\sim 0.45 M_{\odot}$ and thus the peak at $0.3 M_{\odot}$ previously found is absent in this case.

A more detailed analysis of the role played by the red-dwarf population is possible. In Table 2, we summarize the average parameter values of the red-dwarf population for both initial mass functions. Similar sets of data for the white-dwarf population can be found in our previous studies (Camacho et al. 2007; García-Berro et al. 2004). As seen in Table 2, the red-dwarf population constitutes roughly $10 \%$ of the observed MACHO optical depth for a standard initial mass function. It is also important to discuss the other parameters shown in Table 2. For instance, the average mass clearly decreases when the magnitude cut increases, which is the opposite of what occurs for the white-dwarf population. We should expect to find less massive objects for larger magnitude cuts, because the more massive the red dwarf, the brighter it will be. This result is reinforced by the fact that the average distance increases for increasing magnitude cuts. Since the average tangential velocity remains constant, the combined effect a mass that decreases on average and a distance that increases on average is that the Einstein crossing-time remains almost constant. The characteristics of the red-dwarf population differ significantly when the initial mass function of Adams \& Laughlin (1996) is used, since in this case the production of low-mass red dwarfs is heavily suppressed. Accordingly, in our simulations, we did not produce red dwarfs with masses smaller than $\sim 0.45 M_{\odot}$. Thus, since the masses on average are larger, we also find brighter stars. We therefore expect no contribution at all for magnitude cuts above $m_{V} \simeq 22.5^{\mathrm{mag}}$, while for brighter magnitude cuts the average mass expected is $\sim 0.7 M_{\odot}$, which is even larger than expected for $\mathrm{CO}$ white dwarfs.

For the standard initial mass function, a double-peaked profile is found, as already observed; but the peak amplitude, however, should be analyzed in more detail. The ratio of the contribution to the optical depth of a typical red dwarf with respect to the contribution of a typical $\mathrm{CO}$ white dwarf is

$\frac{\tau_{\mathrm{RD}}}{\tau_{\mathrm{CO}}}=\frac{\hat{t}_{\mathrm{RD}}}{\hat{t}_{\mathrm{CO}}} \frac{\varepsilon\left(\hat{t}_{\mathrm{CO}}\right)}{\varepsilon\left(\hat{t}_{\mathrm{RD}}\right)} \approx \sqrt{\frac{M_{\mathrm{RD}} D_{\mathrm{OL}}^{\mathrm{RD}}}{M_{\mathrm{CO}} D_{\mathrm{OL}}^{\mathrm{CO}}}} \frac{\varepsilon\left(\hat{t}_{\mathrm{CO}}\right)}{\varepsilon\left(\hat{t}_{\mathrm{RD}}\right)}$.

This value depends on the adopted initial mass function and on the magnitude cut used. For the standard initial mass function and a magnitude cut of $22.5^{\mathrm{mag}}$, the average values of the mass and distance of a red dwarf are, respectively, $\sim 0.228 M_{\odot}$ and $4.88 \mathrm{kpc}$, while for a typical $\mathrm{CO}$ white dwarf, the average mass is $\sim 0.568 M_{\odot}$ and the average distance is $3.14 \mathrm{kpc}$. For these values, the ratio of the optical depths is equal to $\tau_{\mathrm{RD}} / \tau_{\mathrm{CO}} \approx 0.9$. Thus, although the samples of red dwarfs and white dwarfs differ, their contribution to the optical depth, per object, remains the same. For instance, the red-dwarf population has a smaller average mass than that of white dwarfs but, in contrast, the average distance is larger. The total contribution of both populations is therefore the number of microlenses. To clarify this issue, we evaluated the fraction of microlenses, for the different populations, as a function of the adopted magnitude cut. The results are shown in Fig. 3 for both the standard initial mass function (top panel) and the log-normal initial mass function (bottom panel). For the standard initial mass function, the relative contribution of red dwarfs decreases with increasing value of magnitude cuts, while that of the $\mathrm{CO}$ white dwarfs increases. Both contributions are equal for a magnitude cut of $\approx 24^{\mathrm{mag}}$. Finally, the contribution of $\mathrm{ONe}$ white dwarfs remains roughly constant and only 
Table 2. Summary of the results obtained for the population of red dwarf microlenses towards the LMC for an age of the halo of 14 Gyr, different model initial mass functions, and several magnitude cuts.

\begin{tabular}{lrrrrrrrr}
\hline \hline & \multicolumn{9}{c}{ Standard } & \multicolumn{4}{c}{ AL } \\
\hline Magnitude & 17.5 & 22.5 & 27.5 & 32.5 & 17.5 & 22.5 & 27.5 & 32.5 \\
\cline { 2 - 9 }$\left\langle N_{\mathrm{WD}}\right\rangle$ & $0 \pm 1$ & $0 \pm 1$ & $0 \pm 1$ & $0 \pm 1$ & $0 \pm 1$ & $0 \pm 1$ & $0 \pm 0$ & $0 \pm 0$ \\
$\langle m\rangle\left(M / M_{\odot}\right)$ & 0.324 & 0.228 & 0.119 & 0.092 & 0.747 & 0.622 & - & - \\
$\langle\mu\rangle\left({ }^{\prime \prime} \mathrm{yr}^{-1}\right)$ & 0.018 & 0.011 & 0.006 & 0.005 & 0.010 & 0.002 & - & - \\
$\langle d\rangle(\mathrm{kpc})$ & 2.89 & 4.88 & 8.27 & 10.20 & 5.15 & 17.59 & - & - \\
$\left\langle V_{\mathrm{tan}}\right\rangle\left(\mathrm{km} \mathrm{s}^{-1}\right)$ & 242 & 244 & 254 & 235 & 246 & 180 & - & - \\
$\left\langle\hat{t}_{\mathrm{E}}\right\rangle(\mathrm{d})$ & 41.2 & 48.5 & 41.3 & 43.3 & 98.2 & 158.0 & - & - \\
$\left\langle\tau / \tau_{0}\right\rangle$ & 0.130 & 0.118 & 0.096 & 0.070 & 0.091 & 0.125 & - & - \\
\hline
\end{tabular}

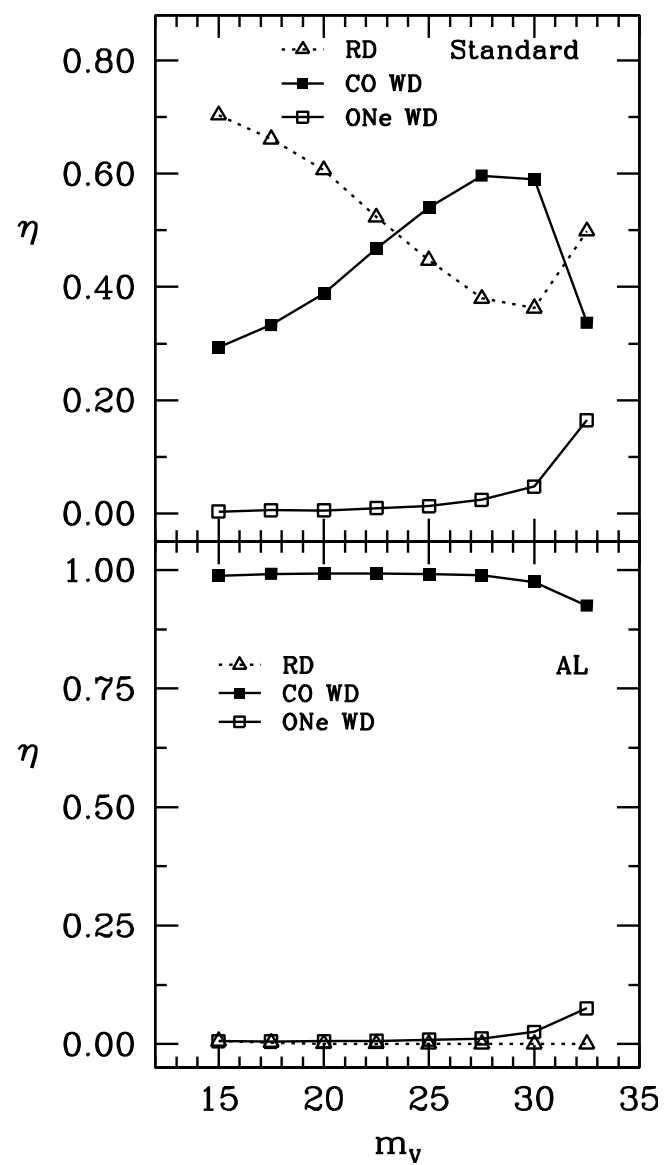

Fig. 3. Fraction of microlenses with respect to the entire population, as a function of the magnitude cut.

becomes significant for large magnitude cuts. These trends can be attributed to the fact that red dwarfs are more numerous at bright magnitudes than white dwarfs, for which typical luminosities are of the order of $\log \left(L / L_{\odot}\right) \simeq-3.5$. The situation is completely different when the log-normal initial mass function of Adams \& Laughlin (1996) is used. As can be seen in the bottom panel of Fig. 3, the number of microlenses is practically dominated by the $\mathrm{CO}$ white-dwarf contribution, while the contribution of red dwarfs and ONe white dwarfs is negligible.

\subsection{The microlensing event rate}

As previously pointed out, the contribution to the optical depth for a standard initial mass function is doubled when the red dwarf population is considered. We have also shown that for a standard initial mass function the contributions of red dwarfs and $\mathrm{CO}$ white dwarfs are roughly the same. It is natural to ask whether there are differences that can help us to discern the contribution of one or another population, using the observational data of the MACHO experiment. To answer this question we analyzed the microlensing rate as a function of event duration. The results of our simulations, for a standard initial mass function, are shown in Fig. 4. Each of the panels is clearly labelled with the adopted magnitude cut and the population of microlenses. In the left panels of Fig. 4, we adopted a magnitude cut of $25^{\mathrm{mag}}$, whilst for the right panels a magnitude cut of $30^{\mathrm{mag}}$ was adopted. In all cases, the simulated microlensing rate is shown using solid lines, while the observational data obtained by the MACHO team is shown using a dotted line. All distributions are normalized to unit area. The red-dwarf and the white-dwarf distributions present some differences. For a magnitude cut of $25^{\mathrm{mag}}$, the white-dwarf population presents a wider distribution, even though both the red-dwarf population and the white-dwarf populations show a peak located at nearly $\sim 20$ days. When a magnitude cut of $30^{\mathrm{mag}}$ is adopted, the differences are more pronounced and it is clear that the distribution peak for the $\mathrm{CO}$ white-dwarf population moves to longer durations ( $\sim 70$ days); in contrast, the peak of the red-dwarf population does not move appreciably.

To be able to attain more quantitative estimates, we performed a $Z^{2}$ statistical test of the compatibility of the different populations with the observed data. The $Z^{2}$ statistical test (Lucy 2000) represents an improvement to the standard $\chi^{2}$ statistical test and was specially developed for small data sets. In Table 3 , we show the $Z^{2}$ probability that the different simulated populations are compatible with the distribution of Einstein times, obtained by the MACHO experiment. It should be clarified that this probability represents an estimate of the degree to which the observed event-rate distribution can be derived from a single population of stars. As can be seen in Table 3, the CO whitedwarf population provides the most appropriate description of the observational data, given that its compatibility is as high as 0.90 for the faintest magnitude cut. Moreover, the compatibility of this population with the observational data increases at fainter magnitude bins. In sharp contrast, the population of red dwarfs presents a decreasing trend as the magnitude cut increases and, additionally, the compatibility with the observational data is, at most, 0.70 . With regard to the ONe white-dwarf population, the compatibility presents an almost constant value of around 0.70 , independently of the magnitude cut. These results indicate that the $\mathrm{CO}$ white-dwarf population can reproduce the observed distribution of microlensing event-rates. Even more, they dominate the behavior of the entire population, as can be seen from the final row of Table 3, in which we analyze the compatibility of the entire population of simulated stars. Therefore, even if the expected number of microlensing events obtained in our 


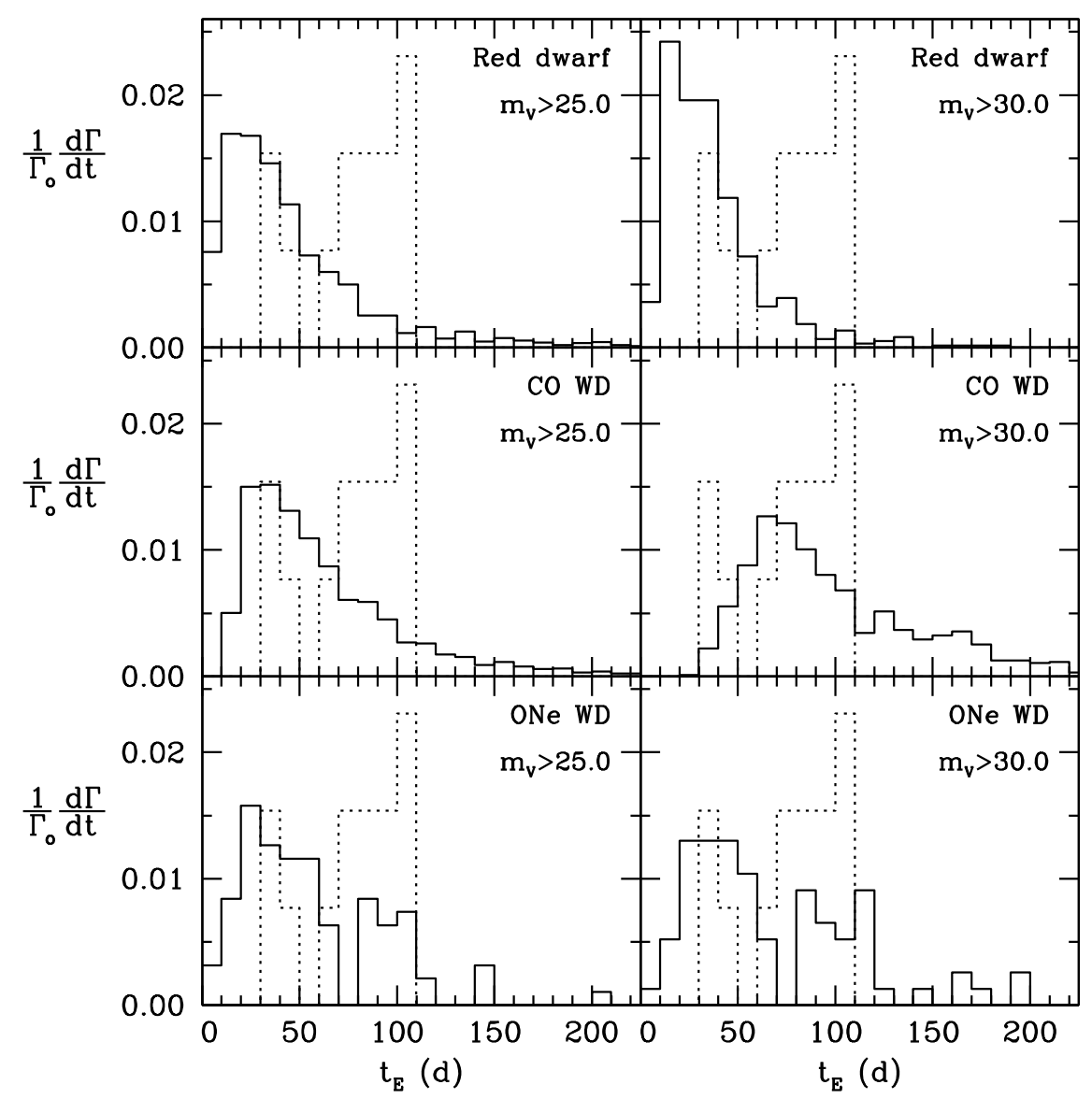

Fig. 4. Differential event rate normalized to unit area as a function of the Einstein crossing-time for the populations under study and different magnitude cuts (solid lines). The observational event distribution from Alcock et al. (2000) is represented as a dotted line in each panel.

Table 3. Compatibility, as obtained using the $Z^{2}$ statistical test, of the observed MACHO distribution of Einstein crossing times and those of the different simulated populations.

\begin{tabular}{lccccc}
\multicolumn{7}{c}{$Z^{2}$ Compatibility test } \\
\hline \hline Magnitude & $17.5^{\mathrm{mag}}$ & $22.5^{\mathrm{mag}}$ & $25.0^{\mathrm{mag}}$ & $27.5^{\mathrm{mag}}$ & $30.0^{\mathrm{mag}}$ \\
\cline { 2 - 6 } Red dwarfs & 0.58 & 0.67 & 0.65 & 0.55 & 0.40 \\
CO white dwarfs & 0.61 & 0.70 & 0.80 & 0.87 & 0.90 \\
ONe white dwarfs & 0.76 & 0.55 & 0.68 & 0.68 & 0.72 \\
Whole population & 0.59 & 0.69 & 0.75 & 0.82 & 0.89 \\
\hline
\end{tabular}

simulations is considerable smaller than the $\sim 15$ events claimed by the MACHO team, the event rate distribution of the $\mathrm{CO}$ white-dwarf population is in fair agreement with the observed distribution. This result places doubt on how well the characteristics of the halo white-dwarf population are known and if there are other ways to produce a larger number of old white dwarfs in the stellar halo.

\subsection{The EROS experiment}

While the MACHO team claim the identification of up to 17 observed events, the EROS collaboration have not found any microlensing event towards the LMC and one candidate event towards the SMC. Adopting a standard halo model and assuming $\tau_{\mathrm{SMC}}=1.4 \tau_{\mathrm{LMC}}$, the EROS results imply an optical depth $\tau_{0}=0.36 \times 10^{-7}$ (Tisserand et al. 2007), which is four times smaller than that obtained by the MACHO team. We performed
Table 4. Summary of the results obtained for the simulation of microlenses towards the LMC for the EROS experiment for an age of the halo of $14 \mathrm{Gyr}$, different model initial mass functions, and several magnitude cuts.

\begin{tabular}{lrrrrrr}
\hline \hline & \multicolumn{3}{c}{ Standard } & \multicolumn{3}{c}{ AL } \\
\hline Magnitude & 17.5 & 22.5 & 27.5 & 17.5 & 22.5 & 27.5 \\
\cline { 2 - 7 }$\left\langle N_{\mathrm{WD}}\right\rangle$ & $0 \pm 1$ & $0 \pm 1$ & $0 \pm 1$ & $1 \pm 1$ & $1 \pm 1$ & $1 \pm 1$ \\
$\langle m\rangle\left(M / M_{\odot}\right)$ & 0.385 & 0.384 & 0.427 & 0.633 & 0.637 & 0.637 \\
$\langle\mu\rangle\left({ }^{\prime \prime} \mathrm{yr}^{-1}\right)$ & 0.020 & 0.013 & 0.009 & 0.028 & 0.022 & 0.011 \\
$\langle d\rangle(\mathrm{kpc})$ & 2.49 & 4.26 & 6.55 & 1.83 & 2.39 & 5.27 \\
$\left\langle V_{\text {tan }}\right\rangle\left(\mathrm{km} \mathrm{s}^{-1}\right)$ & 241 & 269 & 267 & 242 & 250 & 266 \\
$\left\langle\hat{t}_{\mathrm{E}}\right\rangle(\mathrm{d})$ & 38.3 & 45.0 & 54.7 & 42.6 & 50.0 & 75.6 \\
$\left\langle\tau / \tau_{0}\right\rangle$ & 0.558 & 0.695 & 0.163 & 0.839 & 0.628 & 0.488 \\
\hline
\end{tabular}

a set of simulations emulating the conditions of the EROS experiment with inputs similar to those described in Sect. 2. Although only minor differences should be expected in the analysis of the main results, it is clear as well that a joint study of both experiments, using a controlled set of prescriptions, represents a test of the robustness of our numerical procedure.

In Table 4, we summarized the results obtained in this second set of Monte Carlo simulations of microlenses towards the LMC, for the EROS experiment. Our simulations show that, for the standard initial mass function, the expected optical depth could be $70 \%$ of the value found by the EROS team. The value obtained, when only the white-dwarf population was considered, was previously found to be $50 \%$ (Camacho et al. 2007). Our simulations reproduce the results of the EROS experiment more 


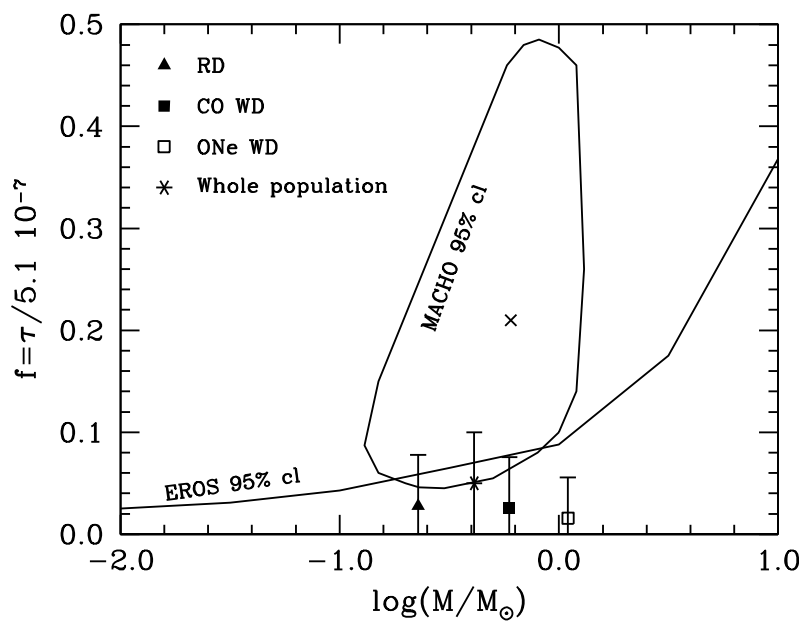

Fig. 5. Halo dark-matter fraction as a function of the mass lens. We plot, using a solid line, the $95 \%$ confidence-level curve for the MACHO experiment and the $95 \%$ confidence-level upper-limit for the EROS experiment.

effectively. The red-dwarf population is obviously responsible for this increase. When a non-standard initial mass function is adopted, the results show, however, only marginal differences with respect to those obtained for a white-dwarf population, given that in this case the role of red dwarfs is limited. In summary, our results are in fair agreement with those obtained by the EROS experiment, and appear to indicate that the microlensing optical depth, obtained by the MACHO collaboration, is an overestimate.

\subsection{The dark matter density}

Using the information presented in this paper, we are able to assess the contribution of stellar populations, for the mass range studied, to the mass of the baryonic dark-matter halo. Based on their $\sim 15$ microlensing events, the MACHO collaboration derived an estimate of the halo fraction of dark matter $f$, as well as the MACHO mass $m$, using maximum-likelihood techniques. A similar analysis was completed by the EROS team, but with the significant difference that, in that case, no event was reported for the LMC, which implies that only an upper limit to the halo mass fraction can be obtained. To compare the results of the MACHO and EROS collaborations with our Monte Carlo simulations, we adopted, as our reference model, the isothermal sphere of core radius $5 \mathrm{kpc}$, with a value of $\rho_{0}=0.0079 M_{\odot} \mathrm{pc}^{-3}$ for the local dark-matter density and disregarding the contribution of the LMC halo. Using this model, we obtained that the optical depth towards the LMC is $\tau_{\mathrm{LMC}}=5.1 \times 10^{-7} f$. The different estimates of the halo mass fraction $f$, as a function of mass, are plotted in Fig. 5. As a solid line we show the curve of the MACHO 95\% confidence level, as taken from Alcock et al. (2000), and the EROS $95 \%$ confidence-level upper-limit, based on no observed events in the EROS-1 and EROS-2 data (Tisserand et al. 2007). We also represent the individual contributions of each population studied and the entire population, in addition to the corresponding $95 \%$ confidence level error bars. It is remarkable that the value obtained for the entire halo simulated-population agrees within the $95 \%$ confidence level curves of both observational estimates. Our results therefore predict that the range of stellar masses within 0.08 and $10 M_{\odot}$, provides $f=0.05$ and an average mass of $0.411 M_{\odot}$ to the halo dark matter, in agreement with the observational data. This result corroborates our previous estimates of the limited contribution of both $\mathrm{CO}$ white dwarfs and ONe white dwarfs (García-Berro et al. 2004; Camacho et al. 2007).

\section{Conclusions}

We have extended our previous studies of the contribution to the halo dark matter of the white-dwarf population and included the Galactic population of red dwarfs. We have estimated the contribution of these objects to the microlensing optical depth towards the LMC and compared our estimate with the measurements of the MACHO and EROS collaborations. Our estimate is based on a series of Monte Carlo simulations that incorporate the most up-to-date evolutionary tracks for red dwarfs, CO white dwarfs, and ONe white dwarfs, and reliable models of our Galaxy and the LMC. In a first set of simulations, we have found that the contribution of the red-dwarf population practically doubles the contribution found so far for the white-dwarf population. Our results indicate that the entire population of these stars can account for at most $\sim 0.3$ of the optical depth found by the MACHO team. This value implies that the contribution of the full range of masses between 0.08 and $10 M_{\odot}$ represents $5 \%$ of the halo dark matter with an average mass of $0.4 M_{\odot}$. Although this result is in partial agreement with the $95 \%$ confidence level MACHO estimate for a standard isothermal sphere and no halo LMC contribution, the expected number of events obtained by our simulations ( 3 events at the $95 \%$ confidence level) is substantially below the 13 to 17 observed MACHO events. These arguments reinforce the idea, previously pointed out by other studies, that the optical depth found by the MACHO team should be an overstimate, probably due to contamination of self-lensing objects, variable stars and others. Moreover, we have assessed the compatibility between the observed event rate distribution and the ones obtained for the different populations under study. Our results show that the $\mathrm{CO}$ white-dwarf population can reproduce fairly well the observed event-rate distribution although, as mentioned earlier, the expected number of events is considerable smaller. On the other hand, the negative results obtained by the EROS team towards the LMC are in agreement with our standard halo simulation. Finally, and for the sake of completeness, we have studied the effects of a log-normal biased initial mass function. In this case, the contribution of the red-dwarf population is only marginal given that the production of low-mass stars is strongly inhibited. Accordingly, the total contribution to the microlensing optical depth is not different from that found in previous studies of the white-dwarf contribution.

Acknowledgements. Part of this work was supported by the MEC grants AYA05-08013-C03-01 and 02, by the European Union FEDER funds and by the AGAUR.

\section{References}

Adams, F. C., \& Laughlin, G. 1996, ApJ, 468, 686

Alcock, C., Allsman, R. A., Alves, D. R., et al. 1997, ApJ, 486, 69

Alcock, C., Allsman, R. A., Alves, D. R., et al. 2000, ApJ, 542, 281

Althaus, L. G., García-Berro, E., Isern, J., Córsico, A. H., \& Rohrmann, R. D. 2007, A\&A, 465, 249

Baraffe, I., Chabrier, G., Allard, F., \& Hauschildt, P. H. 1998, A\&A, 337, 403

Becker, A. C., Rest, A., Stubbs, C., et al. 2005, IAU Symp., 225, 357

Belokurov, V., Evans, N. W., \& Le Du, Y. 2003, MNRAS, 341, 1373

Belokurov, V., Evans, N. W., \& Le Du, Y. 2004, MNRAS, 352, 233

Binney, J., \& Tremaine, H. 1987, Galactic Dynamics (Princeton: Princeton Univ. Press)

Calchi, N. S., de Luca, F., Jetzer, P., \& Scarpetta, G. 2006, A\&A, 459, 407

Camacho, J., Torres, S., Isern, J., Althaus, L. G., \& García-Berro, E. 2007, A\&A, 471,151 
Canal, R., Isern, J., \& Ruiz-Lapuente, P. 1997, ApJ, 488, L35

Chabrier, G. 2004, ApJ, 611, 315

Chabrier, G., Segretain, L., \& Méra, D. 1996, ApJ, 468, 21

Charlot, S., \& Silk, J. 1995, ApJ, 445, 124

Cutri, R. M., Skrutskie, M. F., van Dyk, S., et al. 2003, 2MASS All Sky Catalog of point sources, Univ. of Massachusetts and IPAC/California Institute of Technology

Dehnen, W., \& Binney, J. 1998, MNRAS, 298, 387

Eisenstein, D. J., Liebert, J., Harris, H. C., et al. 2006, ApJS, 167, 40

Finley, D. S., Koester, D., \& Basri, G. 1997, ApJ, 488, 375

Flynn, C., Holopainen, J., \& Holmberg, J. 2003, MNRAS, 339, 817

García-Berro, E., Torres, S., Isern, J., \& Burkert, A. 2004, A\&A, 418, 53

Gates, E. I., \& Gyuk, G. 2001, ApJ, 547, 786

Goldman, B., Afonso, A., Alard, Ch., et al. 2002, A\&A, 389, 69

Griest, K. 1991, ApJ, 366, 412

Gyuk, G., Dalal, N., \& Griest, K. 2000, ApJ, 535, 90

Hambly, N. C., Irwin, M. J., \& MacGillivray, H. T. 2001, MNRAS, 326, 1295

Hansen, B. M. S. 1998, Nature, 394, 860

Hansen, B. M. S., \& Liebert, J. 2003, ARA\&A, 41, 465

Holopainen, J., Flynn, C., Knebe, A., Gill, S. P., \& Gibson, B. K. 2006, MNRAS, 368,1209

Ibata, R. A., Richer, H. B., Gilliland, R. L., \& Scott, D. 1999, ApJ, 524, L95

Iben, I., \& Laughlin, G. 1989, ApJ, 341, 312

Isern, J., García-Berro, E., Hernanz, M., Mochkovitch, R., \& Torres, S. 1998, ApJ, 503, 239

James, F. 1990, Comput. Phys. Commun., 60, 329

Kallivayalil, N., van der Marel, R. P., Alcock, C., et al. 2006, ApJ, 638, 772

Kerins, E., \& Evans, N. W. 1998, ApJ, 503, 75

Kilic, M., Mendez, R. A., Von Hippel, T., \& Winget, D. E. 2005, ApJ, 633, 1126

Klypin, A., Zhao, H., \& Somerville, R. 2002, ApJ, 573, 597

Knox, R. A., Hawkins, M. R. S., \& Hambly, N. C. 1999, MNRAS, 306, 736

Lasserre, T., Afonso, C., Albert, J. N., et al. 2001, A\&A, 355, L39
Liebert, J., Dahn, C. C., \& Monet, D. G. 1989, in White Dwarfs, ed. G. Wegner (Berlin: Springer), 15

Liebert, J., Bergeron, P., \& Holberg, J. 2005, ApJS, 156, 47

Lucy, L. 2000, MNRAS, 318, 92

Majewski, S. R., \& Siegel, M. H. 2002, ApJ, 569, 432

Mollerach, S., \& Roulet, E. 2002, Gravitational Lensing and Microlensing (Singapore: World Scientific)

Muraki, Y., Sumi, T., Abe, F., et al. 1997, ApJ, 490, 493

Nelson, C. A., Cook, K. H., Axelrod, T. S., Mould, J. R., \& Alcock, C. 2002, ApJ, 573, 644

Oppenheimer, B. R., Hambly, N. C., Digby, A. P., Hodgkin, S. T., \& Saumon, D. 2001, Science, 292, 698

Paczyński, B. 1986, ApJ, 304, 1

Rahvar, S. 2005, MNRAS, 356, 1127

Reylé, C., Robin, A. C., \& Créze, M. 2001, A\&A, L53

Sahu, K. 1994, Nature, 370, 275

Salaris, M., García-Berro, E., Hernanz, M., Isern, J., \& Saumon, D. 2000, ApJ, 544,1036

Scalo, J. 1998, in The Stellar Initial Mass Function, ed. G. Gilmore, \& D. Howell (San Francisco: PASP Conf. Ser.), 142, 201

Schneider, P., Kochanek, C. S., \& Wambsganss, J. 2004, in Saas-Fee lectures on Gravitational Lensing

Silk, J. 2007, in The invisible universe: dark matter and dark energy, Proc. of the Third Aegean Summer School (Berlin: Springer Verlag), Lect. Not. Phys. 720, 101

Tisserand, P., Le Guillou, L., Afonso, et al. 2007, A\&A, 469, 387

Torres, S., García-Berro, E., \& Isern, J. 1998, ApJ, 508, L71

Torres, S., García-Berro, E., Burkert, A., \& Isern, J. 2002, MNRAS, 336, 971

Udalski, A., Szymanski, M., Kaluzny, J., et al. 1994, Acta Astron., 44, 1

Vennes, S., Smith, R. J., Boyle, J., et al. 2002, MNRAS, 335, 673

Vidrih, S., Bramich, D. M., Hewett, P. C., et al. 2007, MNRAS, 382, 515

Zhao, H. S. 1998, MNRAS, 294, 139 